\title{
Low-level rf control of Spallation Neutron Source: System and characterization
}

\author{
Hengjie Ma, Mark Champion, Mark Crofford, Kay-Uwe Kasemir, Maurice Piller, Lawrence Doolittle, and Alex Ratti
} (Received 15 December 2005; published 10 March 2006)

\begin{abstract}
The low-level rf control system currently commissioned throughout the Spallation Neutron Source (SNS) LINAC evolved from three design iterations over $1 \mathrm{yr}$ intensive research and development. Its digital hardware implementation is efficient, and has succeeded in achieving a minimum latency of less than $150 \mathrm{~ns}$ which is the key for accomplishing an all-digital feedback control for the full bandwidth. The control bandwidth is analyzed in frequency domain and characterized by testing its transient response. The hardware implementation also includes the provision of a time-shared input channel for a superior phase differential measurement between the cavity field and the reference. A companion cosimulation system for the digital hardware was developed to ensure a reliable long-term supportability. A large effort has also been made in the operation software development for the practical issues such as the process automations, cavity filling, beam loading compensation, and the cavity mechanical resonance suppression.
\end{abstract}

DOI: 10.1103/PhysRevSTAB.9.032001

PACS numbers: 41.20.-q, 07.05.Dz, 02.30.Zz, 01.52.+r

\section{DIGITAL HARDWARE}

The developed field control module (FCM) of Spallation Neutron Source (SNS) LINAC Low-level rf (LLRF) control is in VXI format. It is all digital with 14-bit input and output resolution, and $40 \mathrm{MHz}$ sampling rate. Excluding the peripheral ADCs and DAC, the entire FCM digital hardware is implemented on a single Xilinx field programmable gate array (FPGA) chip XC2V1500, including the direct digital synthesis (DDS) of the IF output. Figure 1 shows the mechanical construction of a FCM. All 96 commissioned LLRF systems are installed in 48 instrument racks distributed along the LINAC Klystron Gallery [1].

\section{A. Development system}

The digital hardware of FCM is modeled in Verilog hardware description language (HDL). The framework and all the required functionalities [2] have been implemented. Currently the Verilog design has 24 components with over 3600 lines of code for synthesis, and over 3000 lines for simulation. The HDL development work by its nature is an ongoing process and the digital hardware will need to continuously evolve and be enhanced in order to adapt to the needs from the commissioning and operation. The ability of efficient and reliable support is equally important as the HDL design itself. For this reason, a great deal of effort was made also in creating a companion software system LLC-suite for providing an integrated development and cosimulation process as shown in Fig. 2. A large part of this process is automated.

The process begins with 2300 lines of handwritten code for synthesis and 1300 lines for the test bench. In each run, the system subsequently machine generates additionally about 1000 lines of synthesis code for the standard functionalities in the design, including the top-level net list, and 2000 lines for the simulation models. The purpose of having an automated machine code generation and simulations is to minimize the risk of human errors. The test suite will continue to grow as more modeling is added into it. The detailed description of this development system, as well as a complete set of FPGA logic and software driver code, is available free at the LLC-suite website authored by Doolittle [3,4].

\section{B. Current implementation}

Figure 3 shows the configuration of the implemented FCM hardware. It is a textbook series $P-I$ controller [5] supported by a CORDIC phase rotator for frequency shifting, one set-point curve table, two feed forward data buffers, five additional data buffers for recording signal traces, and an rf timing sequencer.

The key for a successful implementation of a digital feedback control has been the hardware latency. A careful HDL design for the $P-I$ controller is able to let the signal go through the proportional control path in only 6 clock cycles, which is $150 \mathrm{~ns}$ for the $40 \mathrm{MHz}$ sampling/system clock that the FCM has. Compared with the typical $1 \sim$ $2 \mu$ s total loop delay that SNS LINAC rf system has, the 6 clock cycle latency of the digital hardware has become insignificant, and that allows the use of an all-digital feedback control for the full bandwidth. Another unusual feature with this implementation is that it processes the $I$ and $Q$ signal data in its original sequential format throughout the entire process in a single channel, resulting in minimal hardware and minimum latency.

The implemented essential functionalities also include four history data buffers for simultaneously recording four vector signal traces selectable from the forward and reflected wave of the rf power, cavity field, and LLRF output drive, plus an extra data buffer for study. The history buffers have the standard ZOOM/PAN feature of a digital storage oscilloscope (DSO) for variable time resolutions and starting points. The DSO feature proved essential for the system development, troubleshooting, and operation automation. There are two switched 8-kByte feed forward 


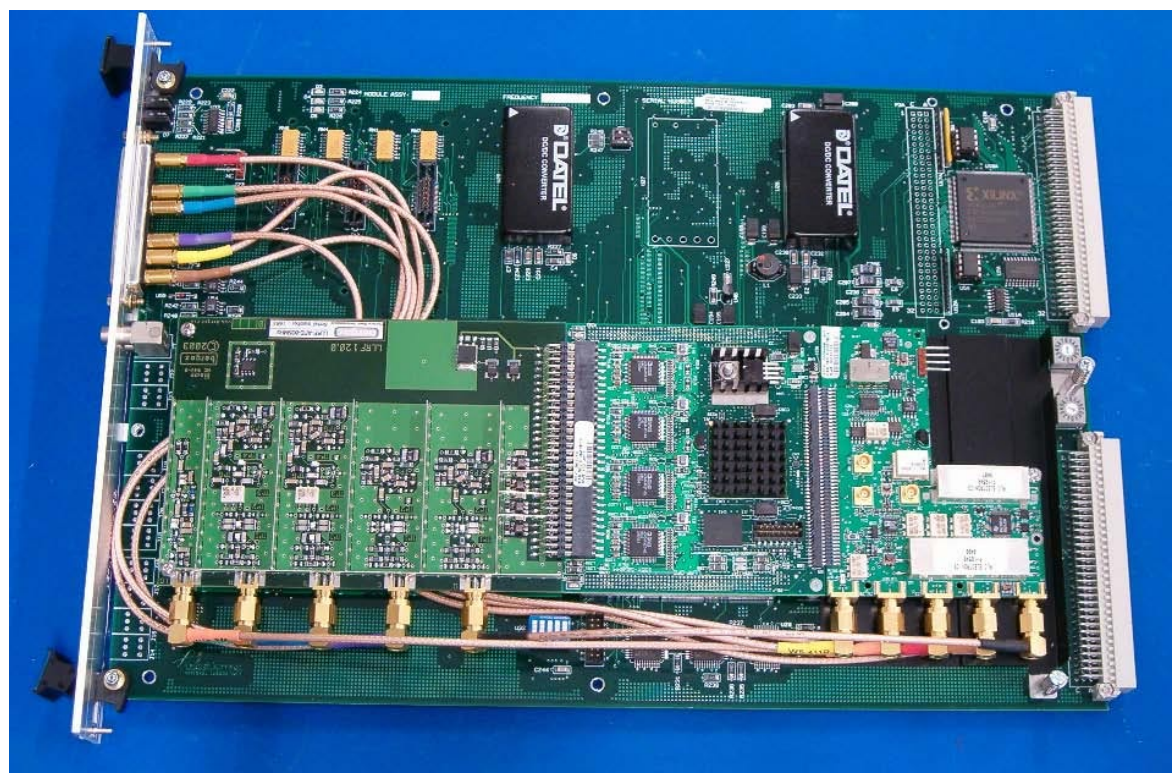

FIG. 1. (Color) FCM of SNS LINAC LLRF is built with a VXI carrier and three daughter-cards: analog front end in the left, digital front end (DFE) in the middle and rf output in the right. The core of this unit is DFE which performs all the control functions.

data buffers, one for the rf drive waveform of cavity filling, and another for the beam loading compensation. There is also a 1-kByte long table for the set-point curve. A CORDIC algorithm based phasor rotator is also coded in the HDL [6]. The rotator spins the set-point phasor to shift the output frequency up to $+/-645 \mathrm{kHz}$. The function of the output frequency shift is needed during the cavity tuning or heating. The phase reference signal data is collected in a time slot either prior to or after the rf pulse. That allows the reference signal to come into the system either

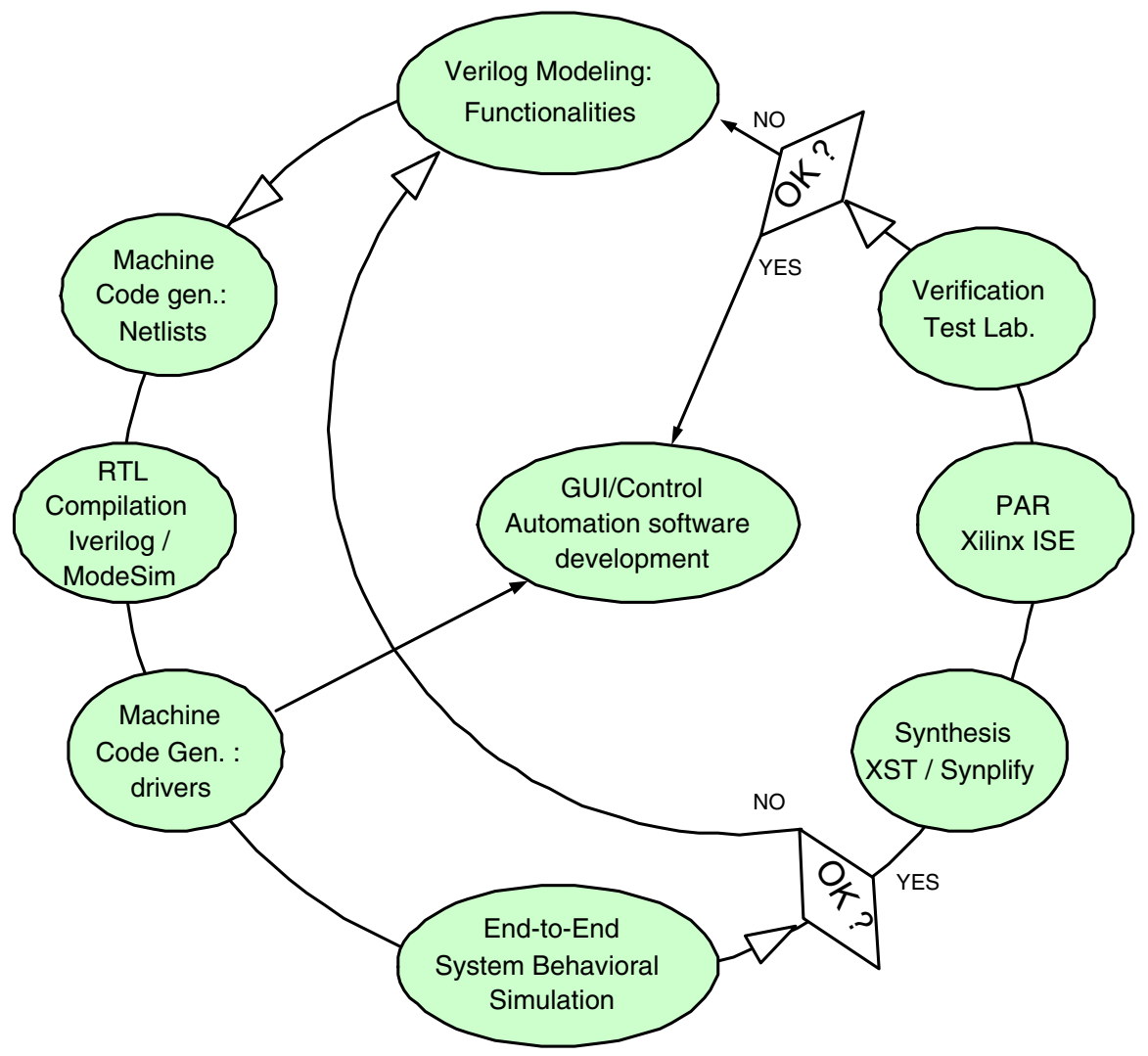

FIG. 2. (Color) Verilog HDL modeling and cosimulation process for the development of FCM digital hardware. 


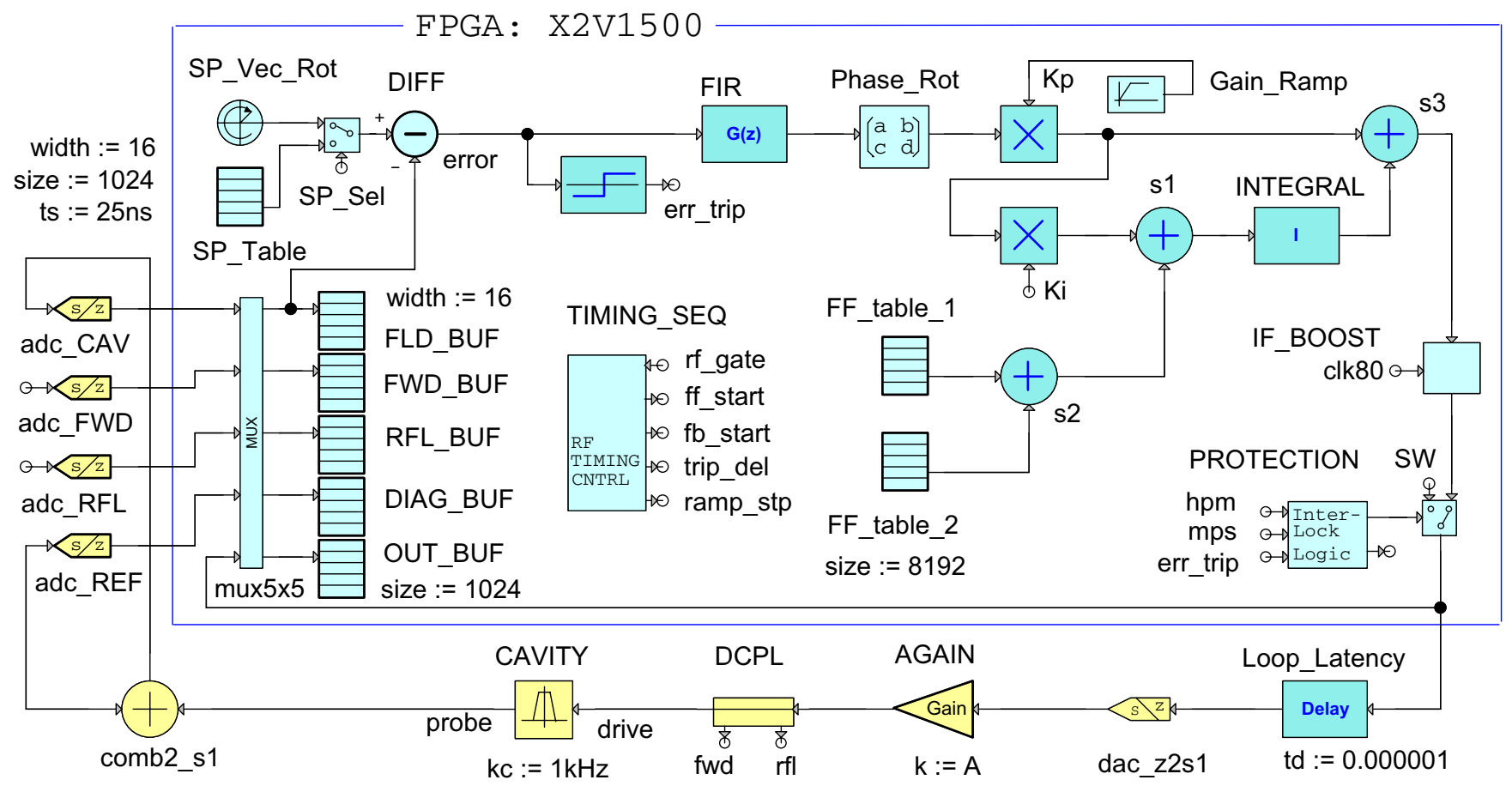

FIG. 3. (Color) A model of SNS rf control system. All required control functionalities in the blue box are implemented in one FPGA chip.

through a dedicated channel or through the channel timeshared with the cavity signal [7].

After the place-and-route, the entire design has used about $20 \%$ of the logic slices, $30 \%$ of the RAMBs, $50 \%$ of the IOBs, and $8 \%$ of the multipliers of the XC2V1500 FPGA chip.

\section{SYSTEM CHARACTERIZATION}

\section{A. Frequency response}

A system analysis at the cavity probe where the end-toend point in the control loop is defined can be conveniently carried out in continuous time. The CPI $805 \mathrm{MHz}$ klystron used in the SNS LINAC exhibits a fairly good linearity around the normal operating point. If we can ignore the Lorentz force detuning effect on the basis of small-signal analysis, we may assume a simplistic 1st-order model for the cavity which is perfectly on its resonance [8]. Under these assumptions, we may first examine the theoretical limits posed by the loop delay on the loop gain and consequently on the control bandwidth through a linear timeinvariant (LTI) system analysis. The open-loop transfer function is expressed in (1) as

$$
\begin{aligned}
G(s) & =\mathbf{K}_{\mathbf{p}} \cdot \mathbf{g} \cdot\left(1+\frac{K_{i}}{s}\right) \cdot \frac{\omega_{b}}{s+\omega_{b}} \cdot \frac{\omega_{b p f}}{s+\omega_{b p f}} \cdot e^{-\tau \cdot s} \\
& \approx \mathbf{g} \cdot \mathbf{K}_{\mathbf{p}} \cdot \omega_{b} \cdot \frac{\left(s+K_{i}\right)}{s\left(s+\omega_{b}\right)} \cdot e^{-\tau \cdot s}, \text { when } \omega_{b} \ll \omega_{b p f}
\end{aligned}
$$

where $\omega_{b}$ and $\omega_{b p f}$ are the half-bandwidth of the cavity and the output bandpass filter of the LLRF. The $\tau$ is the total loop delay. $\mathbf{K}_{\mathbf{p}}$ is the digital proportional gain produced inside the $P-I$ controller, while $\mathbf{g}$ is the lumped-sum gain along the analog path of the feedback loop outside of the controller. Therefore, the product $\mathbf{g} \cdot \mathbf{K}_{\mathbf{p}}$ represents the total loop gain. The scalar $K_{i}$ is the integral gain.

The effect of the Klystron bandwidth is not included in the transfer function as the klystron bandwidth is as wide as $3 \mathrm{MHz}$, and has an insignificant effect for the bandwidth of analysis. The above open-loop transfer function indicates a Type 1 , and minimum $(n+2)$ th-order system, where the number $n$ is the order of the Padé approximation for the delay term.

For a unity feedback, the closed-loop transfer function therefore is

$$
\begin{aligned}
G B(s) & =\frac{G(s)}{1+G(s)} \\
& =\frac{\mathbf{g} \cdot \mathbf{K}_{\mathbf{p}} \cdot \omega_{b}\left(s+K_{i}\right) \cdot e^{-\tau \cdot s}}{s\left(s+\omega_{b}\right)+\mathbf{g} \cdot \mathbf{K}_{\mathbf{p}} \cdot \omega_{b}(s+K i) \cdot e^{-\tau \cdot s}} .
\end{aligned}
$$

The added poles from the loop delay $\tau$ have created the possibility for instability to occur, and limited the maximum loop gain that can be used, and that in turn has limited the control bandwidth and precision. To analytically examine the stability of such a system, we may first simplify the closed-loop transfer function (2) by setting $\mathbf{K}_{\mathbf{i}}=\boldsymbol{\omega}_{\mathbf{b}}$ to cancel the cavity pole, and substitute the delay term with a 3rd order Padé approximation. That renders a characteristic function 


$$
\begin{aligned}
F(s)= & s\left(1+\frac{1}{4} \tau \cdot s\right)+\mathbf{g} \cdot \mathbf{K}_{\mathbf{p}} \cdot \omega_{b} \cdot\left(1-\frac{3}{4} \tau \cdot s\right. \\
& \left.+\frac{1}{4}(\tau \cdot s)^{2}-\frac{1}{24}(\tau \cdot s)^{3}\right) .
\end{aligned}
$$

Using Routh-Hurwitz criterion, we obtain a condition for the system stability approximately as

$$
\mathbf{g} \cdot \mathbf{K}_{\mathbf{p}} \cdot<\frac{4}{3 \cdot \tau \cdot \omega_{b}} .
$$

This result shows how the maximum total loop gain is limited by the total loop delay. It also shows the system with a narrower cavity bandwidth allows a greater loop gain. A major part in the total loop delay is from the signal transmission cables and rf waveguides. Their lengths are dictated by the machine and building layouts. RouthHurwitz stability criterion is only necessary, but not sufficient, and therefore, the limit of loop gain predicted by the expression (4) may not always be achievable. Another factor that affects the accuracy of the prediction is the order of Padé approximation used for obtaining (4). A lower 3rd-order approximation is used here for illustrating the limiting effect of the loop delay and cavity bandwidth on the maximum total loop gain that can be used. A higher order approximation as used next will give a much closer result.

The system analysis for stability and performance can also be conveniently carried out with a graphic method. Figure 4 is a Nichols chart plotted using the ideal system transfer function as expressed in (1), and a sixth-order Padé approximation for the delay term. Assuming a worst case of $2 \mu \mathrm{s}$ total loop delay, the chart indicates that the system with a normal-conducting (NC) cavity of $10 \mathrm{kHz}$ halfbandwidth will become unstable (gain margin is reduced to zero) as the value of loop gain reaches 12 , while the limit for the system with a superconducting (SC) cavity of $550 \mathrm{~Hz}$ bandwidth would be 210. The amount of loop gain for an optimal damping condition for the same SC cavity is 75 . For the SC cavity and a loop gain of 75 , the open-loop transfer function curve intercepts the $-3 \mathrm{~dB}$ line at the frequency of $95 \mathrm{kHz}$, and that indicates the $3 \mathrm{~dB}$ closed-loop control bandwidth of this system. The phase and gain margin are also found to be about $60^{\circ}$ and $8 \mathrm{~dB}$, respectively. The phase margin reduces to zero at $115 \mathrm{kHz}$. From this chart, the predicted critically dampened control bandwidth is $95 \mathrm{kHz}$ even under the worst case for the loop delay. However, the actually measured result (to be described in the following section) is about only $60 \mathrm{kHz}$. The discrepancy may be caused by some of important factors such as the Lorentz detuning of the cavity that are not included in the simplified system analysis model.

Finding out the limit of usable loop gain is important because the loop gain is a primary determinant of the control bandwidth and error. In the case of the FCM, it is a $P-I$ controller (with an integral term in its transfer function), and therefore is a type- 1 system. That means that in the case of the normal-conducting cavities that have the time constants far smaller than the rf pulse width, the value of the loop gain affects only the closed-loop control

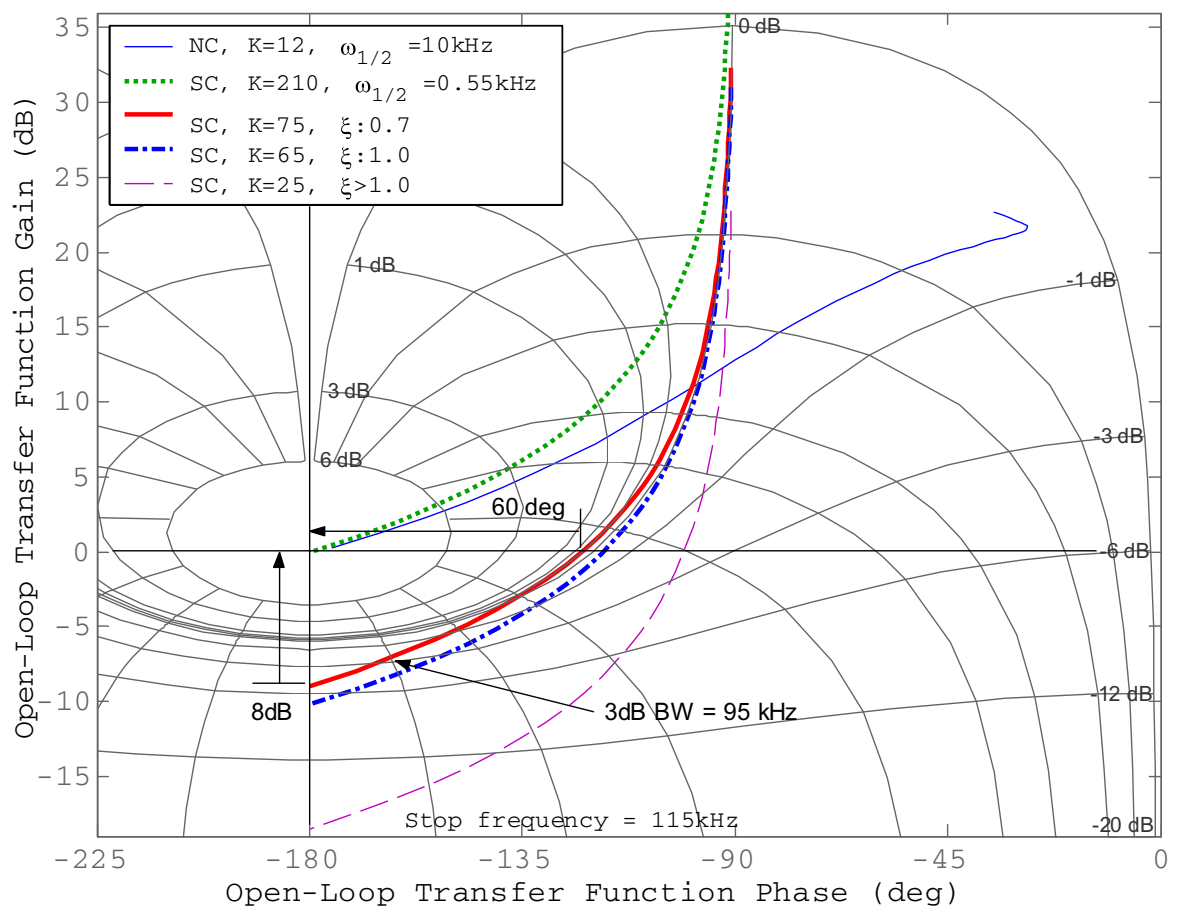

FIG. 4. (Color) Graphical analysis with Nichols chart for the performance and stability of SNS rf control system. A worst case of $2 \mu \mathrm{sec}$ loop delay is assumed. 
bandwidth, but not the control error as it becomes zero after the system reaches a steady state. Contrary to the NC cavities, the superconducting cavities have the time constants comparable to the rf pulse width. The control system cannot reach a steady state within the duration of the rf pulse. In a transient state, the amount of loop gain affects not only the control bandwidth in the form of responding time, but also the control error. The Nichols chart analysis indicates that in the case of SNS SC cavities, the maximum loop gain that can be used without causing instability is 210 , while the optimal value is about 75 . Based on control theories, with only the feedback control and the gain of 75 , the estimated maximum field error would be about $1.3 \%$ in the case of $100 \%$ beam loading, which exceeds the specified precision of $0.5 \%$. Because of that, the help of a feed forward compensation is necessary for achieving the required control precision for SC cavities.

\section{B. Transient response}

On the commissioned NC cavities, the transient response was measured with a commonly used method for a $P-I$ controller. A test step is added to the amplitude set point, and the response at the cavity field probe is observed. The choice of end-to-end point is consistent with the one used in the previous section. The size of the step was $12.5 \%$ of the nominal field level. The systems were tuned up by matching the time constant of the controller integrator to that of the cavity for a zero-pole cancellation. Figure 5 shows the test result on drift tube linac (DTL) Tank 1 with a cavity bandwidth of $16 \mathrm{kHz}$. The result shows the control system reaches to a critically dampened condition when the loop gain is at about 10 , and the corresponding closedloop control bandwidth in baseband is about $166 \mathrm{kHz}$. This result is close to the predicted value.

Figures 6 and 7 show the transient response tested on a superconducting cavity system SCL-12a. The test method used on SC cavities was slightly different from that for NC cavities. The $50 \mu$ s rectangular pulse was injected into the loop from the feed forward signal input, and the response was observed at the cavity rf input port. The choice of endto-end point is different, but the test is equivalent, and so the test results for the two types of cavities can be comparable. The pulse amplitude is $20 \%$ of the normal rf drive level. The high-beta SC cavity SCL-12a has a halfbandwidth of $560 \mathrm{~Hz}$, and operates at a field gradient of $9 \mathrm{MV} / \mathrm{m}$. A maximum loop gain of 99 was measured for the system to reach a slightly under damping ratio of 0.78 , and achieved $3-\mathrm{dB}$ control bandwidth is about $56 \mathrm{kHz}$. The measured value of the control bandwidth is lower than the $95 \mathrm{kHz}$ predicted in the Nichols chart.

The transient response to a $10 \mathrm{~mA}, 300 \mu$ s unchopped beam pulse was also observed on the high-beta superconducting cavity SCL-12a as shown in Figs. 8 and 9. The test beam pulse had an about $10 \mu$ s rise time on its leading edge. With a high loop gain of 89 and without any feed forward compensation, the beam loading produced an initial peak error under $0.3 \%$ on the cavity field amplitude, and the error then reduced to less than $0.1 \%$ after about $30 \mu \mathrm{s}$.

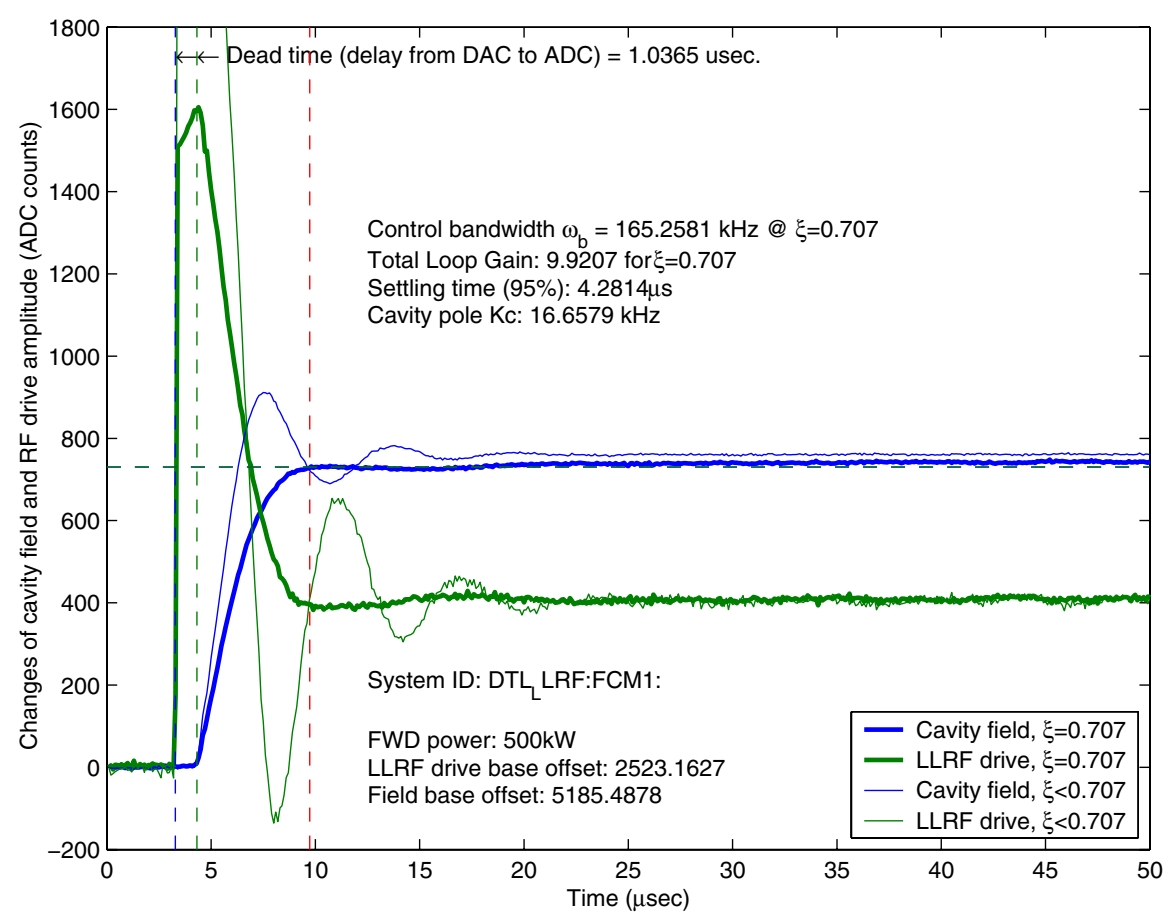

FIG. 5. (Color) Transient response of FCM field control on NC cavity DTL-1 to a $12.5 \%$ step in the amplitude set value. The measurement result shows a typical behavior of a 2nd order LTI feedback control system. 


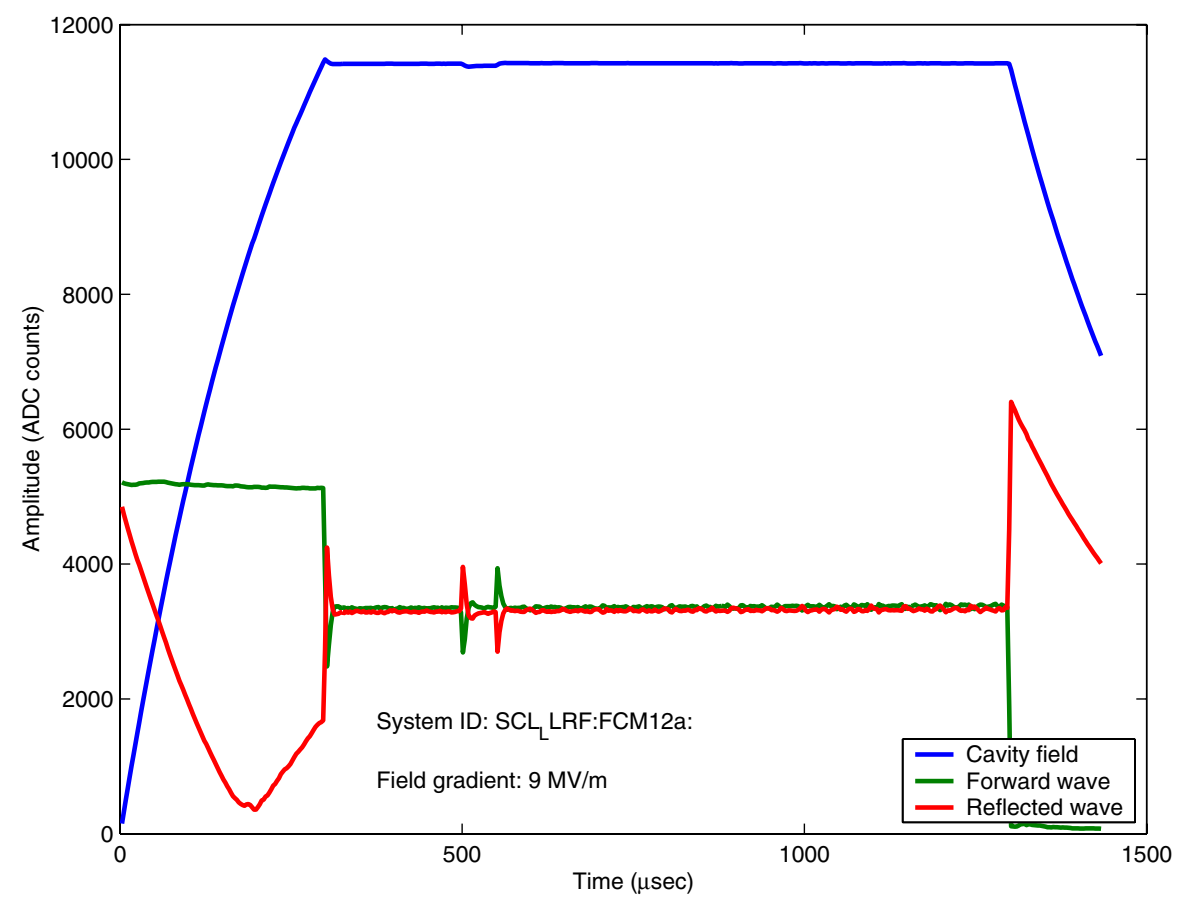

FIG. 6. (Color) A full view of the FCM transient response to a $20 \%$ step in feed forward input, measured on superconducting cavity SCL-12a.

\section{ISSUES WITH CONTROLLING SUPERCONDUCTING CAVITIES}

\section{A. Mechanical mode of SC cavities}

SNS superconducting cavities have various mechanical resonance modes. The medium-beta cavities typically have a mechanical mode at about $2 \mathrm{kHz}$, excited by the ponderomotive force of the pulsed $\mathrm{rf}$ drive. The resonating mode modulates the cavity field phase in open-loop control, and in closed-loop control, the modulation on the field phase is also coupled onto the amplitude. The modulation on the cavity field during the flattop is mostly visible on the

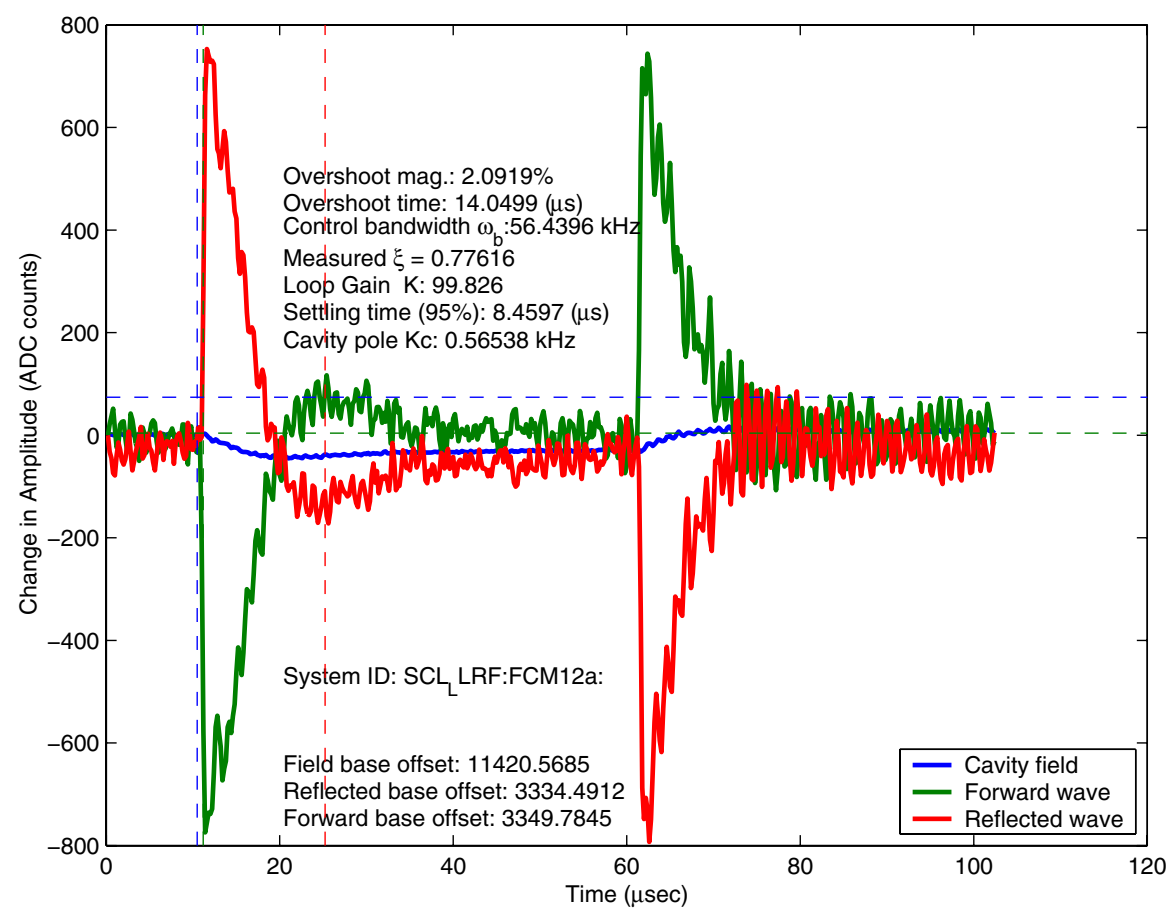

FIG. 7. (Color) A detailed view of the FCM step response measured on superconducting cavity SCL-12a. 


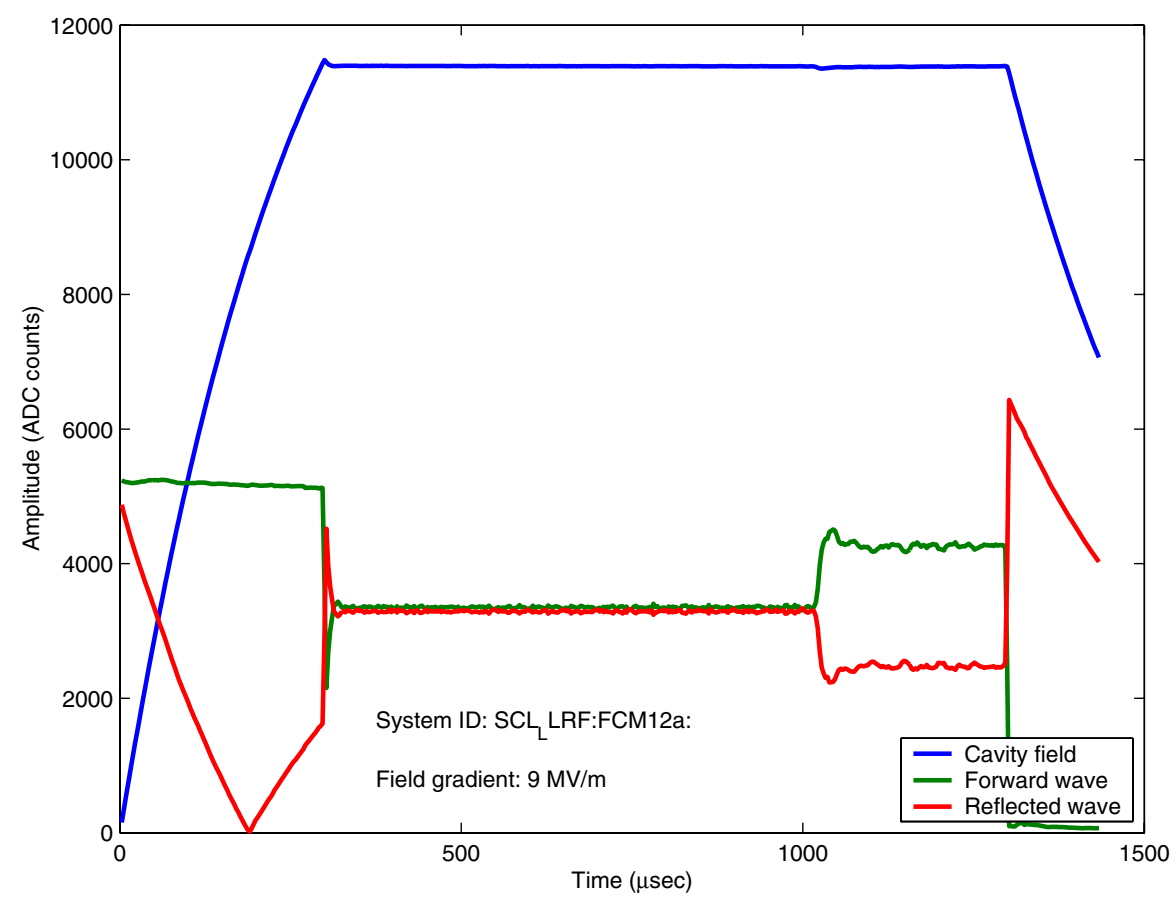

FIG. 8. (Color) A full view of the FCM transient response to a $10 \mathrm{~mA}, 300 \mu \mathrm{s}$ beam pulse on superconducting cavity SCL-12a. The beam loading occurs at the end of the rf pulse.

medium-beta cavities, and it is usually very small when those cavities are operated at the designed $10 \mathrm{MV} / \mathrm{m}$ field gradient. But it becomes prominent when the field gradient greatly exceeds the designed level. An example of such an effect is illustrated by the test result on cavity SCL-7A as shown in Fig. 10.

A moderate loop gain of 41 (vs maximum allowed 83) was used for this test. During the commissioning, it was

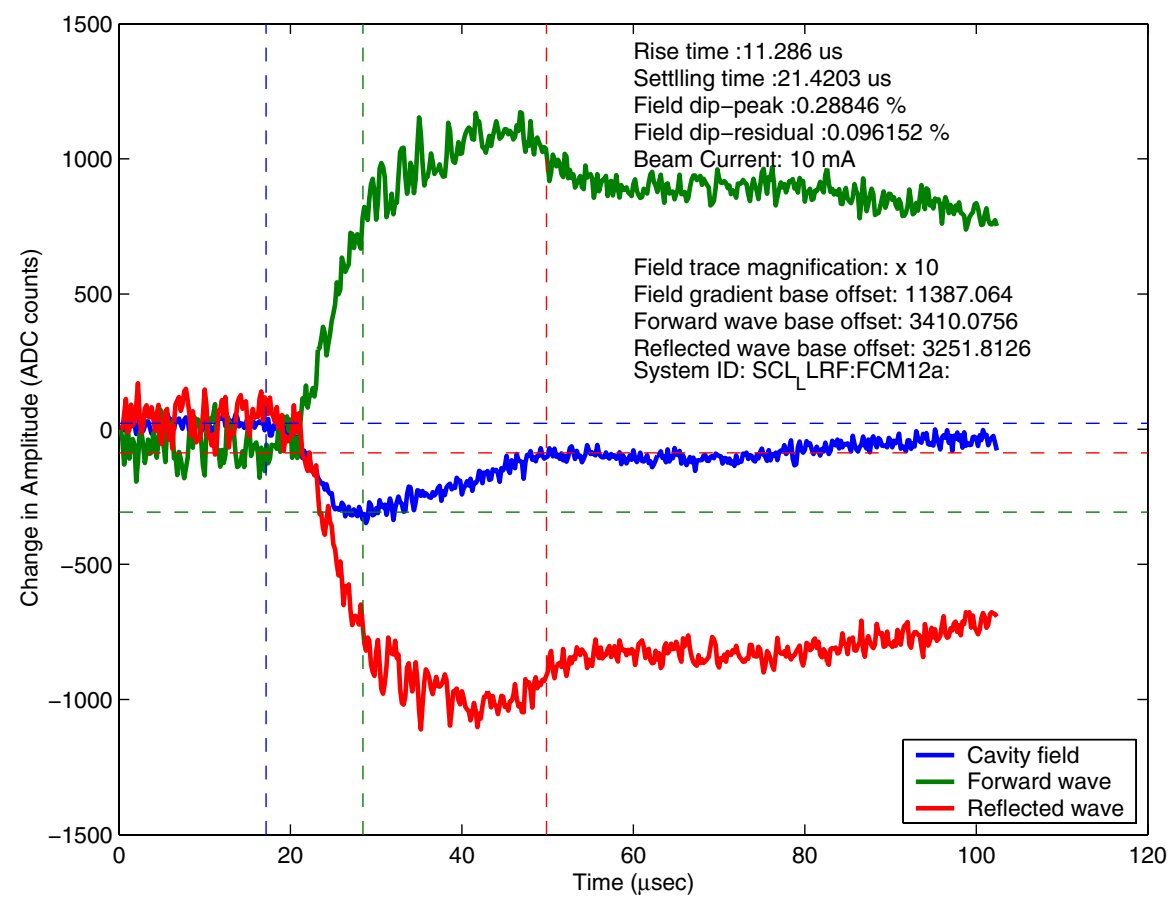

FIG. 9. (Color) A detailed view of the FCM transient response to a $10 \mathrm{~mA}, 300 \mu$ s beam on superconducting cavity SCL-12a. The cavity field trace is magnified by an extra factor of 10 so that the beam loading becomes visible. 


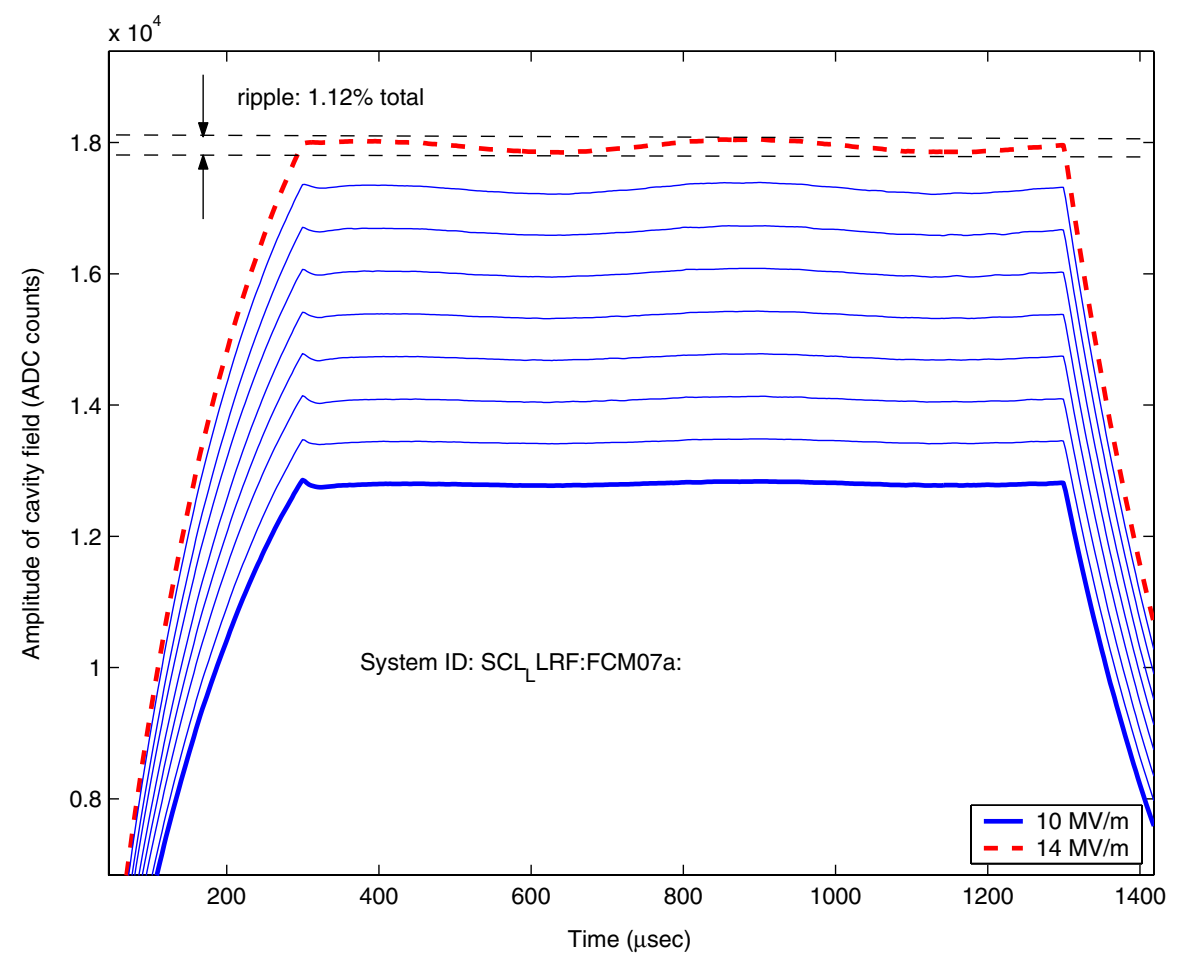

FIG. 10. (Color) The magnitude of $2 \mathrm{kHz}$ mechanical mode grows as the cavity operating field gradient increases beyond the designed $10 \mathrm{MV} / \mathrm{m}$ level. The measurement was done on SC cavity SCL-07a, and a moderate loop gain of 41 was used.

decided that many of the medium-beta cavities would need to operate at the field gradient levels much beyond the design value, and therefore the damping of this mechanical mode becomes necessary. From the testing, it has been found that both strong feedback control and feed forward compensation are effective for the damping.

Figure 11 shows the change in the magnitude of the mode on the field amplitude and phase as the loop gain
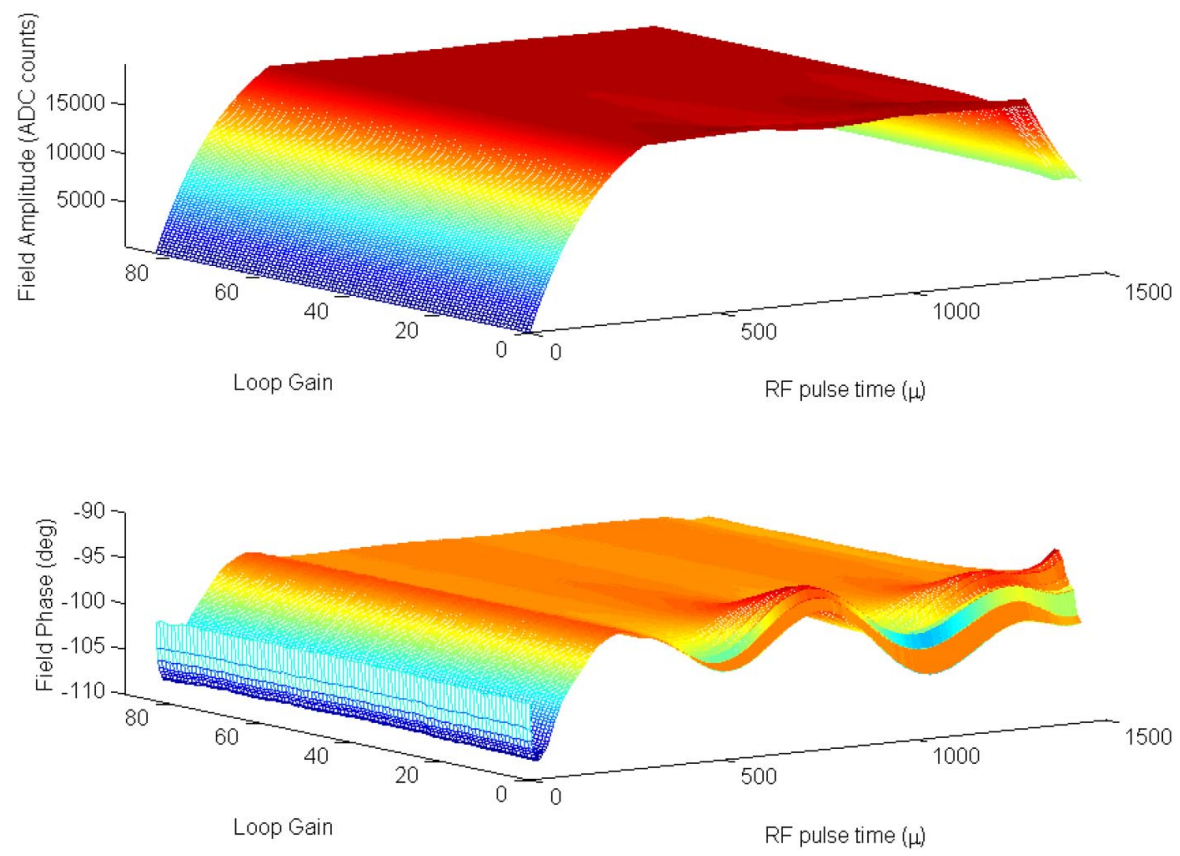

FIG. 11. (Color) The ripples of the mechanical mode on the cavity field amplitude and phase are largely damped out as the loop gain of the feedback control reaches sufficiently high. In this measurement, the value of the loop gain is varied from zero (i.e. no feedback control) to 83 over 100 rf pulses. 
increase from 0 to 83 on cavity SCL-7a under test. At the maximum loop gain of 83 , the magnitude of the $2 \mathrm{kHz}$ wave on the field amplitude is reduced to $0.56 \%$, peak to peak. It is interesting that when there is no feedback control (i.e. zero loop gain), the mode manifests itself mainly on the field phase. But once the feedback is turned on, the mode starts to transfer to the field amplitude.

Another test on the same cavity was also conducted to prove the effectiveness of the mechanical mode suppression using an adaptive feed forward (AFF) compensation. Figure 12 shows the result. With an optimal loop gain of 83 , the initial magnitude of ripple on the field due to the mode was about $0.5 \%$ in amplitude, and $0.5^{\circ}$ in phase, peak to peak, before the compensation starts. After only 9 iterations, the adaptive feed forward was able to reduce the error to less than $0.1 \%$ in the amplitude and $0.1^{\circ}$ in the phase. The AFF is surprisingly effective in suppressing this $2 \mathrm{kHz}$ mechanical mode.

\section{B. 5/6 $\pi$ mode in cavity field}

From the early time of the designing for the LINAC, there had been a concern about the excitation of $5 / 6 \pi$ mode in the field by the chopping of the beam. The frequency of this mode is about $800 \mathrm{kHz}$ from the $805 \mathrm{MHz}$ center frequency, and there is a possibility that this harmonic frequency may affect the field control. The $5 / 6 \pi$ mode can be excited by the chopped beam pulses and the switching edges of the rf pulses. Using the digital oscilloscope function PAN/ZOOM of the history buffers

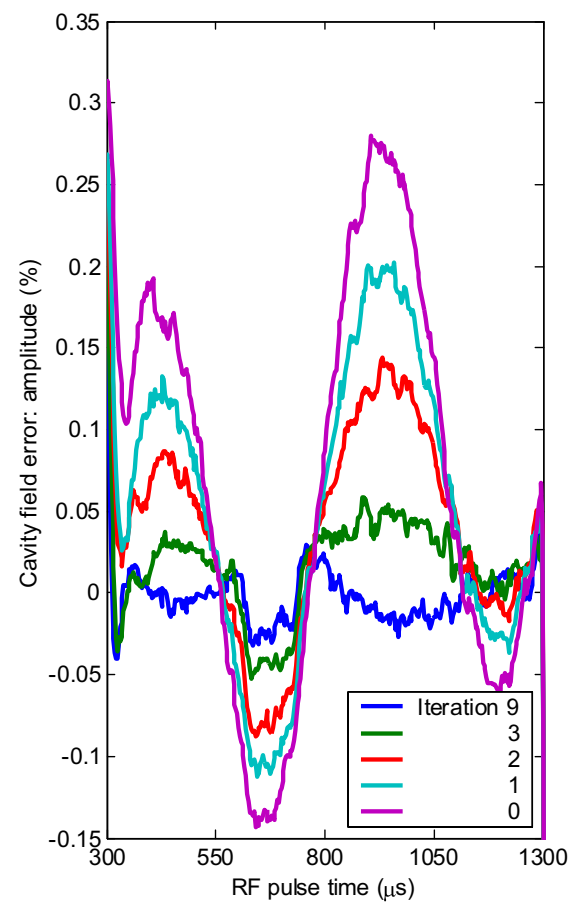

in the FCM, we are able to observe the details of this $5 / 6 \pi$ mode excited by the rf over the duration of rf pulse. Figure 13 shows an example of such an observation made on a high-beta cavity SCL-13a running in closedloop control mode, while Fig. 14 shows the same measurement made when the system is running in open-loop mode.

The waterfall spectral histograms are generated by performing FFT analysis on the field data of a $25 \mu$ s window moving across the $1300 \mu \mathrm{s}$ rf on time axis in 40 steps, one step per pulse. The sampling rate is $5 \mathrm{MHz}$. The magnitude of the spectrum is in the $Z$ axis, while the frequency is in the $X$ axis. The $Y$ is the time axis which covers the time from the beginning to the end of the rf pulse. Both plots show that the $800 \mathrm{kHz} 5 / 6 \mu$ mode started at the moment that the rf is switched on and also at the moment of switching off. At the moment when the cavity filling is completed and the flat top begins, the magnitude of this mode has decayed well below $1 \%$ of the primary mode (the absolute scale is not shown on the normalized spectra). Based on this observation, it may be concluded that the excitation of the $5 / 6 \mu$ mode has not posed a big problem to the operation in the case of macro beam pulse (unchopped beam). Further study is needed for the case of chopped beam pulse.

\section{OPERATION METHODS}

The FCM has a high control bandwidth and has demonstrated good control stability. For NC cavities which have relatively small time constants, the FCM allows an option

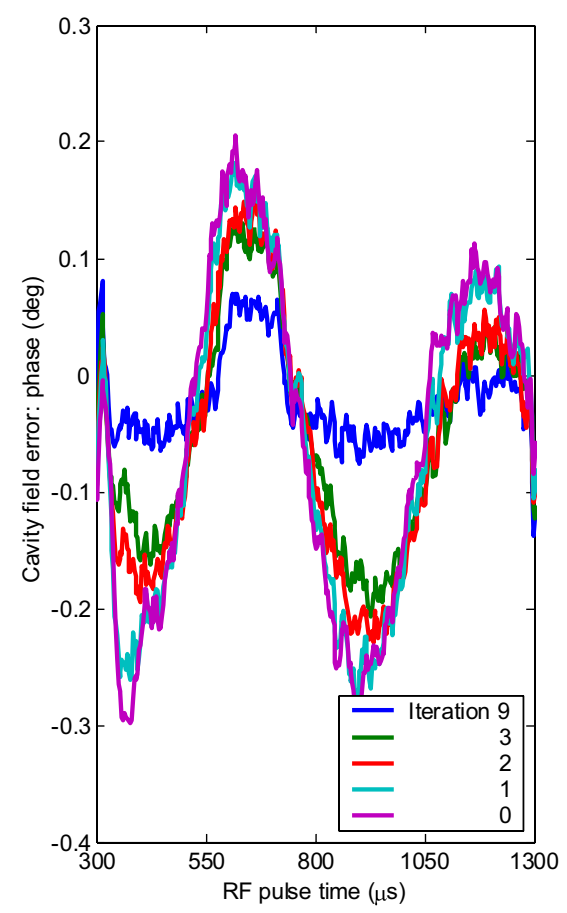

FIG. 12. (Color) The adaptive feed-forward compensation is also a very effective means for damping the mechanical mode. The pulseto-pulse field error minimizing is a basic adaptive correction process with the feed forward compensation vector reiteratively modified by a sum of the proportion and integral of the field error vector, and each of the two terms in the sum is individually scaled. 


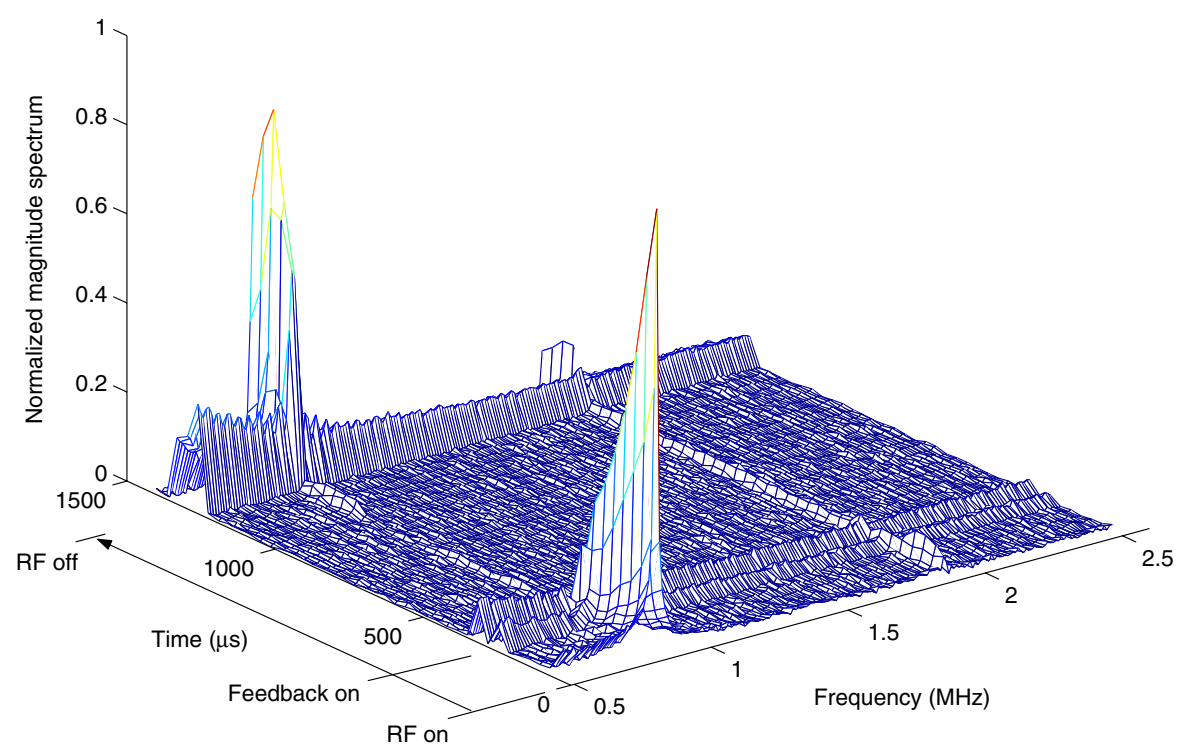

FIG. 13. (Color) $5 / 6 \pi$ mode excited by the switching of rf power. The measurement was made on cavity SCL-13a running in closedloop control.

of running the cavities in a simplistic feedback control only mode all the time without the assistance of a feed forward drive for cavity filling. At the beginning of each rf pulse, the system in closed-loop mode can quickly get over the transient state and reach a steady state with a zero control error (because of the nature of the type- 1 system) in much less than $100 \mu \mathrm{s}$. Given the total rf pulse width of $1300 \mu \mathrm{s}$, it leaves a flattop more than required $1000 \mu \mathrm{s}$ for the beam. The undesirable behavior of large-signal response during the initial transient time can be greatly modified by using set-point curves.
The operation of the SC cavities does require a procedure of an initial cavity filling so that the following closedloop control during the flat top will be able to operate in a small-signal region. For this reason, an automated LLRF operation procedure has been developed and being used on the commissioned LINAC. The screen picture in Fig. 15 shows some of the details of this operation mode.

In the waveform windows, the blue trace is the signal of Klystron incident wave to the cavity, and the green trace is for the reflected wave. The red trace is the cavity field. Given the maximum rf pulse of $1300 \mu \mathrm{s}$, and the designed

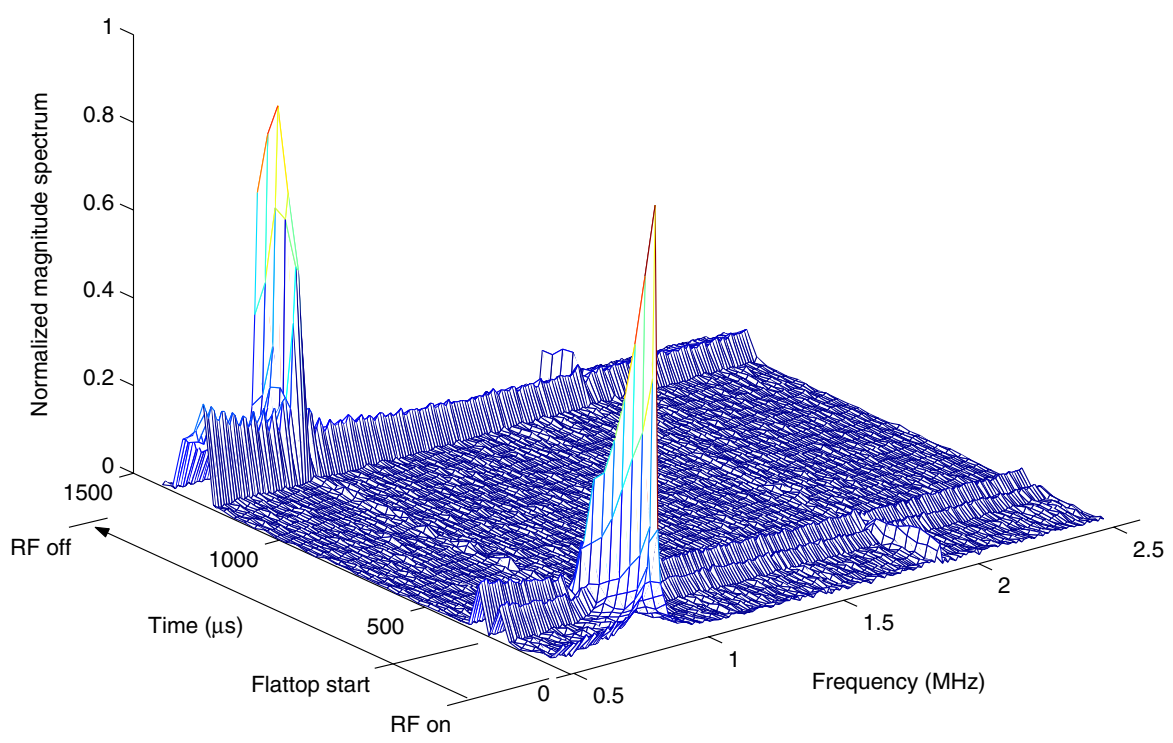

FIG. 14. (Color) $5 / 6 \pi$ mode excited by the switching of $\mathrm{rf}$ power. The measurement was made on SC cavity SCL-13a running with no feedback control (open loop). 


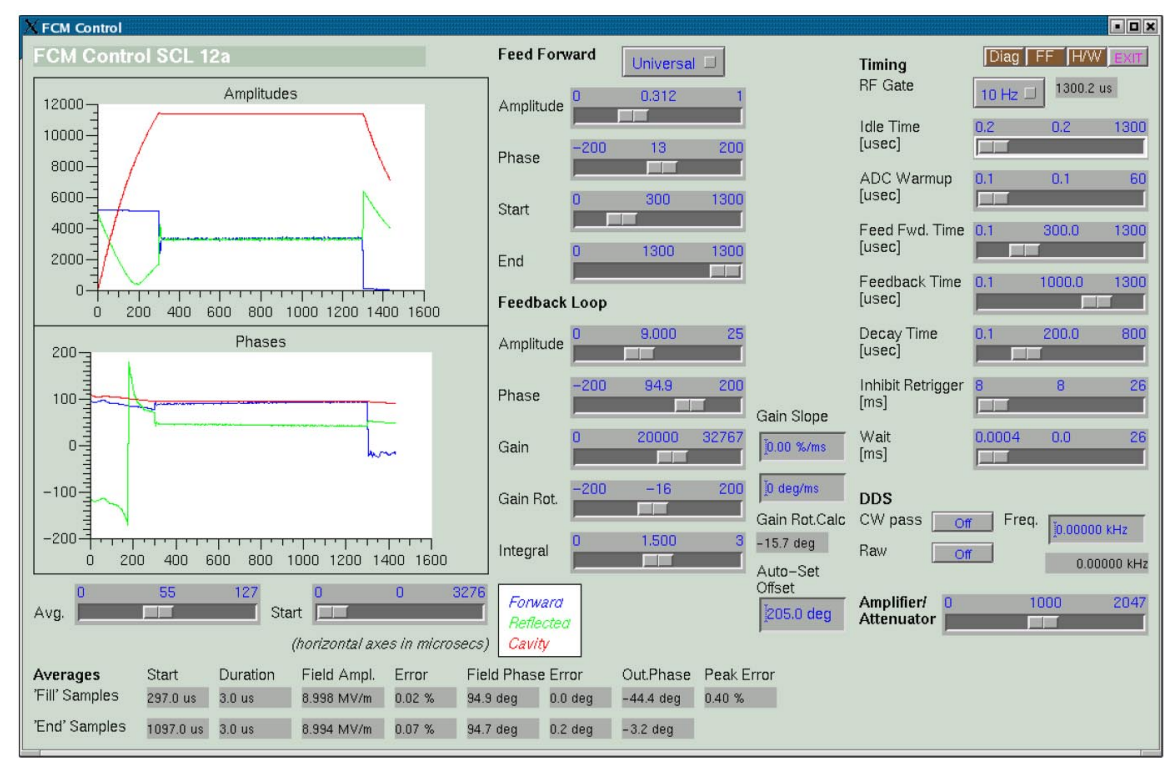

FIG. 15. (Color) Current operation method of the FCM for cavity filling and field regulation.

beam pulse width of $1000 \mu$ s for SNS LINAC, the maximum cavity filling time allowed is $300 \mu \mathrm{s}$. The procedure starts with a cavity filling for $300 \mu$ s in open-loop control mode. The rf drive power is higher during this period of time to make the cavity field rise exponentially to the target level in the given $300 \mu \mathrm{s}$. At the end of filling time, the cavity field amplitude and phase need to be very close to the set values for the subsequent closed-loop control. The software measures the field level and phase and makes adjustments for the next pulse. At the $300 \mu$ s time mark, the open-loop rf drive power reduces to a lower level for holding the cavity field at this level for the remaining period of the pulse. The waveforms for the Klystron forward power and cavity field in Fig. 15 clearly illustrate this pattern. The feedback control is also turned on at the $300 \mu$ s time mark and starts regulating the cavity field over the period of flat top. The gain slope hardware feature provides the capability to ramp the loop gain from a low value to the full strength in a specified short time period. The purpose is to ease the transition at the moment when the system control changes from open-loop to closed-loop mode. The waveform data for the cavity filling is stored in the primary feed forward (FF) data buffer, and is played out on every rf pulse. There is also a secondary FF data buffer for the adaptive feed forward compensation for the beam loading. The secondary FF buffer is turned on only when the beam is present. The work in the adaptive feed forward compensations will be described in details in the future reports.

\section{DISCUSSION}

During the development of SNS LLRF control system, much knowledge and experience related to cavity rf control in general have been gained. Some of the important issues specific to the digital hardware development have also been understood. Those issues include the common concerns in the latency of the digital hardware, the linearity of the front end, the noise and shielding, as well as the clock distribution. The importance of the capability of the cosimulation process for the developed digital hardware has been specially appreciated. The cavity rf system with a digital control is a mixed-signal system. An effective cross-domain simulation is therefore necessary for studying the behavior of the developed rf control system. Specifically, that requires that the actual register transfer level synthesis code of the digital logic and the math-based analog models for the rest of the rf system be simulated together. The effort in that direction will continue for SNS rf control system.

\section{ACKNOWLEDGMENTS}

The SNS LLRF project is a collaborated effort among ORNL, LBNL, and LANL. Here the authors would like to acknowledge the contributions from every team member. SNS is managed by UT-Battelle, LLC, under Contract No. DE-AC05-00OR22725 for the U.S. Department of Energy. SNS is a partnership of six national laboratories: Argonne, Brookhaven, Jefferson, Lawrence Berkeley, Los Alamos, and Oak Ridge

[1] M. Champion, in Proceedings of the Particle Accelerator Conference, Knoxville, TN, 2005 (IEEE, Piscataway, NJ, 2005).

[2] M. Champion, Report No. SNS 104010300-SR0002-R00, 2002. 
[3] L. Doolittle, llc-suite, http://recycle.lbl.gov/llc-suite/

[4] L. Doolittle, LBNL Technical Note No. FE-EE-021, 2001, FE3000.

[5] B. Kuo, Automated Control Systems (Prentice-Hall, Englewood Cliffs, NJ, 1987).
[6] L. Doolittle, LBNL posted Technical Note, 2002; http:// recycle.lbl.gov/ ldoolitt/plan50MHz/

[7] M. Piller, in Proceedings of the Particle Accelerator Conference, Knoxville, TN, 2005 (Ref. [1]).

[8] T. Czarski, TESLA Report No. 2003-08. 